\title{
Assessment of the Gradient of an Objective Function by Analytical Derivation for Optimization-Based Design of Ground-Coupled Heat Pump Systems
}

Bernard Dusseault

\begin{abstract}
Optimization-based design of ground heat exchangers requires derivation of the objective function with respect to the design parameters, which is usually done through finite-differentiation of the cost or utility function. The approach is however prone to approximation errors and can result in convergence issues or long optimization time. By deriving analytically the ground heat exchanger transfer function, it is possible to obtain an exact representation of the objective function gradient and avoid numerical instabilities. To illustrate the advantages of using analytical expressions, a common design task is expressed as an optimization problem. It is shown that by using an analytical derivation of the gradient in conjunction with strong Wolfe conditions during a line search may reduce significantly computation time by comparison to a finite-differentiation of the gradient.
\end{abstract}

\section{INTRODUCTION}

Two approaches are commonly used to design a ground-coupled heat pump system. The first one relies on sizing equations, like the one suggested by ASHRAE (Kavanaugh and Rafferty, 2014; Philippe et al., 2010; Bernier et al., 2008), while the second approach consists of iteratively using a simulation method to optimize a cost or utility function. The optimization process can be achieved by trial and error, which can be cumbersome, or automated through nonlinear optimization algorithms (Retkowski and Thöming, 2014; Huang et al., 2015; Hénault et al., 2016). The latter however requires the computation of the derivative of the objective function with respect to the $n$ design parameters in order to find a suitable descent direction.

The objective function gradient is usually computed by finite difference through $n+1$ simulations of the ground-coupled heat pump system. Although being easy to implement, computation of objective function gradient through finite differentiation often leads to inaccurate estimations of the descent direction, which may significantly increase solution time. The objective of this paper is to present two efficient computational approaches to derive the gradient of an objective function corresponding to a common design task encountered by designers.

Bernard Dusseault (bernard.dusseault@polymtl.ca) is a PhD student, Philippe Pasquier and Denis Marcotte are professors at Polytechnique Montréal. 


\section{METHODOLOGY}

For a ground heat exchanger (GHE) composed of $n_{b}$ boreholes of length $H$, the mean fluid temperature $\bar{T}_{f}$ circulating in the boreholes can be described by:

$$
\bar{T}_{f}(t)=T_{g}+\frac{\dot{Q}}{H n_{b}} R_{b}+(f * g)(t)
$$

where $T_{g}$ is the initial ground temperature, $\dot{Q}$ is the total ground load exchanged by the GHE and $R_{b}$ is the equivalent borehole resistance (Gehlin, 2002; Marcotte and Pasquier, 2008). The last term represents the temperature perturbation at the borehole wall and is obtained by convolving the incremental heat flux signal $f$ given by:

$$
f\left(t_{i}\right)=\frac{\dot{Q}\left(t_{i}\right)}{H n_{b}}-\frac{\dot{Q}\left(t_{i-1}\right)}{H n_{b}}
$$

with the response function $g$ (Eskilson, 1987). Note that as indicated by Marcotte and Pasquier (2008) and Pasquier and Marcotte (2013), for $i=0$ in Eq. 2, a zero value should be used for $\dot{Q}\left(t_{i-1}\right)$.

\section{Construction of the Transfer Function $g$}

The GHE response function $g$ synthetizes the thermal behavior of the GHE and integrates the thermal properties of the underground, but also the coordinates and length of the boreholes composing the GHE. Although many methods can be used to construct $g$, the approach chosen in this article is based on the work of Marcotte and Pasquier (2014) because of its efficiency and flexibility. The approach, itself inspired by the works of Lamarche (2009), Cimmino et al. (2013) and Lazzarotto (2014), consists to find sequentially the heat transfer rates $\mathbf{q}_{t}$ emanating from the boreholes of a GHE using a linear system of equations expressed, for simplicity, in a compact matrix notation. For a parallel arrangement of the boreholes, the system corresponding to the mean temperature at the borehole wall is given by:

$$
\left[\begin{array}{cc}
\mathbf{G} & \mathbf{1} \\
\mathbf{1}^{T} & 0
\end{array}\right]\left[\begin{array}{c}
\mathbf{q}_{\mathbf{t}} \\
-\Delta \widetilde{T}_{b}
\end{array}\right]=\left[\begin{array}{c}
-\mathbf{h} \\
\mathbf{1}
\end{array}\right]
$$

where $\mathbf{q}_{t}$ is a $n_{b} \times 1$ vector containing the heat load emitted by each borehole for the current time step, $\mathbf{h}$ is a $n_{b} \times 1$ vector containing the historical temperature perturbations, $\mathbf{1}$ is a $n_{b} \times 1$ vector of ones and $G$ is the following interaction matrix:

$$
\mathbf{G}=\left[\begin{array}{cccc}
G_{11} & G_{12} & \cdots & G_{1 n_{b}} \\
G_{21} & G_{i j} & & \\
\vdots & & \ddots & \\
G_{n_{b} 1} & & & G_{n_{b} n_{b}}
\end{array}\right]
$$

In this work, matrix $G$ is constructed using the finite line-source (FLS) model of Claesson and Javed (2011) with a heating load of $1 \mathrm{~W} / \mathrm{m}$ at radial distances $r_{i j}$ and at an evaluation time corresponding to the current time step value. Thus, the $G_{i j}$ are given for a borehole of length $H$, thermal diffusivity $\alpha$ and buried depth $D$ by

with

$$
G_{i j}=\frac{1}{4 \pi k} \int_{1 / \sqrt{4 \alpha t}}^{\infty} e^{-r_{i j}{ }^{2} s^{2}} \frac{Y(H s, D s)}{H s} \mathrm{~d} s
$$


Before solving Eq. 3 for $\mathbf{q}_{t}$ and $\Delta \widetilde{T}_{b}$, the historical vector $\mathbf{h}$ has to be built. For time step $m$, the latter is obtained by solving the following convolution product at $t=m \Delta t$ :

$$
\mathrm{h}_{i j}(m \Delta t)=\left(\tilde{f}_{j} * \tilde{g}\right)(m \Delta t)
$$

where the incremental heat load vector for borehole $j$ is given by:

$$
\tilde{f}_{j}\left(t_{i}\right)=q_{j}\left(t_{i}\right)-q_{j}\left(t_{i-1}\right)
$$

and where the transfer function $\tilde{g}$ is the borehole response for the first $m$ time steps obtained under a unit load for each $r_{i j}$ radial distance. Since $b$ has to be calculated at each time step, Eq. 7 must be solved for $\Delta \widetilde{T}_{b}$ sequentially as well by using all the previous values of $\mathbf{q}_{t}$ and $\mathbf{G}$. Finally, the transfer function corresponding to the mean temperature at the borehole wall used in Eq. 1 is obtained simply by:

$$
g(t)=n_{b} \Delta \widetilde{T}_{b}(t)
$$

\section{A Simple Objective Function}

A common task performed by GHE designers consists to identify the total underground loop length keeping the fluid temperature within the heat pump operation range and to keep the GHE cost as low as possible. If the building is a heating dominant one, the total loop length $\left(n_{b} H\right)$ chosen by the designer should be sufficient to maintain the fluid temperature just above the heating temperature limit $\left(T_{\min }\right)$ of the heat pump, while minimizing, for a fixed $n_{b}$ value, the borehole length. From an optimization perspective, such situation is easily described by an objective function describing the gap between the minimum fluid temperature given by Eq. 1 and $T_{\min }$ through:

$$
F=\left(\min \left(\bar{T}_{f}(t)\right)-T_{\min }\right)^{2}
$$

Since $F$ is a function of $\mathrm{R}_{b}, H, n_{b}, T_{\min }$ and the borehole coordinates, minimizing $F$ is in fact a multidimensional problem. For the sake of keeping this case study simple and instructive, only the borehole length $H$ is used as design parameter, thus allowing to reduce the problem to a unidimensional one with a well-defined global minimum. The next section will now focus on finding the gradient of this objective function according to $H$ with three different approaches.

\section{Analytical derivation of the gradient}

The gradient of $F$ with respect to the design parameter is obtained by computing the derivative of $F$ with respect to $H$. This leads to:

$$
\frac{\partial F}{\partial H}=2\left(\min \left(\bar{T}_{f}(t)\right)-T_{\min }\right) \frac{\partial \min \left(\bar{T}_{f}(t)\right)}{\partial H}
$$

To evaluate the derivative of $T_{f}$ according to $H$, Eq. 1 should be derived as well. Now, dropping the min and notation to simplify the writing, the derivative is evaluated at the time during where the minimum in the fluid temperature occurs:

$$
\frac{\partial \min \left(\bar{T}_{f}(t)\right)}{\partial H}=\frac{-\dot{Q}}{H^{2} n_{b}} R_{b}+\left(\frac{\partial f}{\partial H} * g\right)(t)+\left(f * \frac{\partial g}{\partial H}\right)(t)
$$

with

$$
\frac{\partial f}{\partial H}=\frac{-\dot{Q}\left(t_{i}\right)}{H^{2} n_{b}}-\frac{-\dot{Q}\left(t_{i-1}\right)}{H^{2} n_{b}}
$$


and

$$
\frac{\partial g}{\partial H}=n_{b} \frac{\partial \Delta \widetilde{T}_{b}}{\partial H}
$$

Now, the derivative of the transfer function with respect to $H$ is needed to solve Eq. 12 and it is necessary to derive Eq. 3 as well. Differentiating Eq. 3 and solving for $\partial \Delta \widetilde{T}_{b} / \partial H$ (see Petersen and Pedersen, 2012) leads to:

$$
\left[\begin{array}{c}
\partial \mathbf{q}_{\mathbf{t}} / \partial H \\
-\partial \Delta \widetilde{T}_{b} / \partial H
\end{array}\right]=-\left[\begin{array}{cc}
\mathbf{G} & \mathbf{1} \\
\mathbf{1} & 0
\end{array}\right]^{-1}\left[\begin{array}{cc}
\partial \mathbf{G} / \partial H & \mathbf{0} \\
\mathbf{0} & 0
\end{array}\right]\left[\begin{array}{cc}
\mathbf{G} & \mathbf{1} \\
\mathbf{1} & 0
\end{array}\right]^{-1}\left[\begin{array}{c}
-\mathbf{h} \\
\mathbf{1}
\end{array}\right]+\left[\begin{array}{cc}
\mathbf{G} & \mathbf{1} \\
\mathbf{1} & 0
\end{array}\right]^{-1}\left[\begin{array}{c}
-\partial \mathbf{h} / \partial H \\
\mathbf{0}
\end{array}\right]
$$

Since all the terms in G were obtained using the FLS model of Cleasson and Javed (2011), their formulation has to be derived as well according to $H$ and this gives:

$$
\frac{\partial G_{i j}}{\partial H}=\frac{1}{4 \pi k} \int_{1 / \sqrt{4 \alpha t}}^{\infty} e^{-r_{i j}^{2} s^{2}}\left(\frac{Y(H s, D s)}{H^{2} s^{2}}+\frac{Z(H s, D s)}{H s}\right) \mathrm{d} s
$$

with

$$
Z(h, d)=2 \operatorname{erf}(b)+2 \operatorname{erf}(b+2 d)-2 \operatorname{erf}(2 h+2 d)
$$

Finally, Eq. 15 to 17 are solved sequentially to construct $\partial g / \partial H$ which requires computation of

$$
\frac{\partial \mathrm{h}_{i j}}{\partial H}(m \Delta t)=\left(\tilde{f}_{j} * \partial G_{i j} / \partial H\right)(m \Delta t)
$$

\section{Finite-differentiation of the gradient}

The finite-difference method involves a simple and well known algorithm commonly used for the calculation of gradients. In this article, a forward finite difference scheme is used to approximate the derivative of the objective function $F$ according to $H$ between $H$ and $H+\varepsilon$ :

$$
\frac{\partial F}{\partial H}=\frac{F(H+\varepsilon)-F(H)}{\varepsilon}
$$

\section{Semi-analytical derivation of the gradient}

Evaluating sequentially Eq. 15 to 18 can be cumbersome and to avoid this task, an hybrid approach relying both on finite-difference and analytical derivation is also presented. The semi-analytical derivation of Eq. 10 consists to solve Eq. 3 for $\Delta \widetilde{T}_{b}$ with $H$ and $H+\varepsilon$ to evaluate $\partial \Delta \widetilde{T}_{b} / \partial H$ (Eq. 14) by finite difference through:

$$
\frac{\partial \Delta \widetilde{T}_{b}}{\partial H}=\frac{\Delta \widetilde{T}_{b}(H+\varepsilon)-\Delta \widetilde{T}_{b}(H)}{\varepsilon}
$$

Once the solution of Eq. 20 is obtained, Eq. 11 to 14 are solved to obtain the objective function gradient. Note that the terms involving $\dot{Q}\left(t_{i}\right) / H n_{b}$ are derived analytically. We will show that with this approach, the implementation is simpler but still retains the precision of the analytical approach. 


\section{A SIMPLE CASE STUDY}

To assess the precision of the gradient computed by the three methods presented earlier, Eq. 1 has been used to simulate over a 10 year-period the mean fluid temperature circulating in a GHE made of 25 boreholes embedded in a soil having a ground thermal conductivity $k$ of $2.5 \mathrm{~W} / \mathrm{mK}$, a thermal diffusivity $\alpha$ of $1.25 \mathrm{e}-6 \mathrm{~m}^{2} / \mathrm{s}$ and a ground temperature $T_{g}$ of $10{ }^{\circ} \mathrm{C}$. The boreholes have an equivalent resistance $R_{b}$ of $0.1 \mathrm{mK} / \mathrm{W}$, are located on a regular grid with spacing of 10 and $5 \mathrm{~m}$ at a depth $D$ of $2 \mathrm{~m}$. The ground loads illustrated in Figure 1 were used to solve Eq. 1
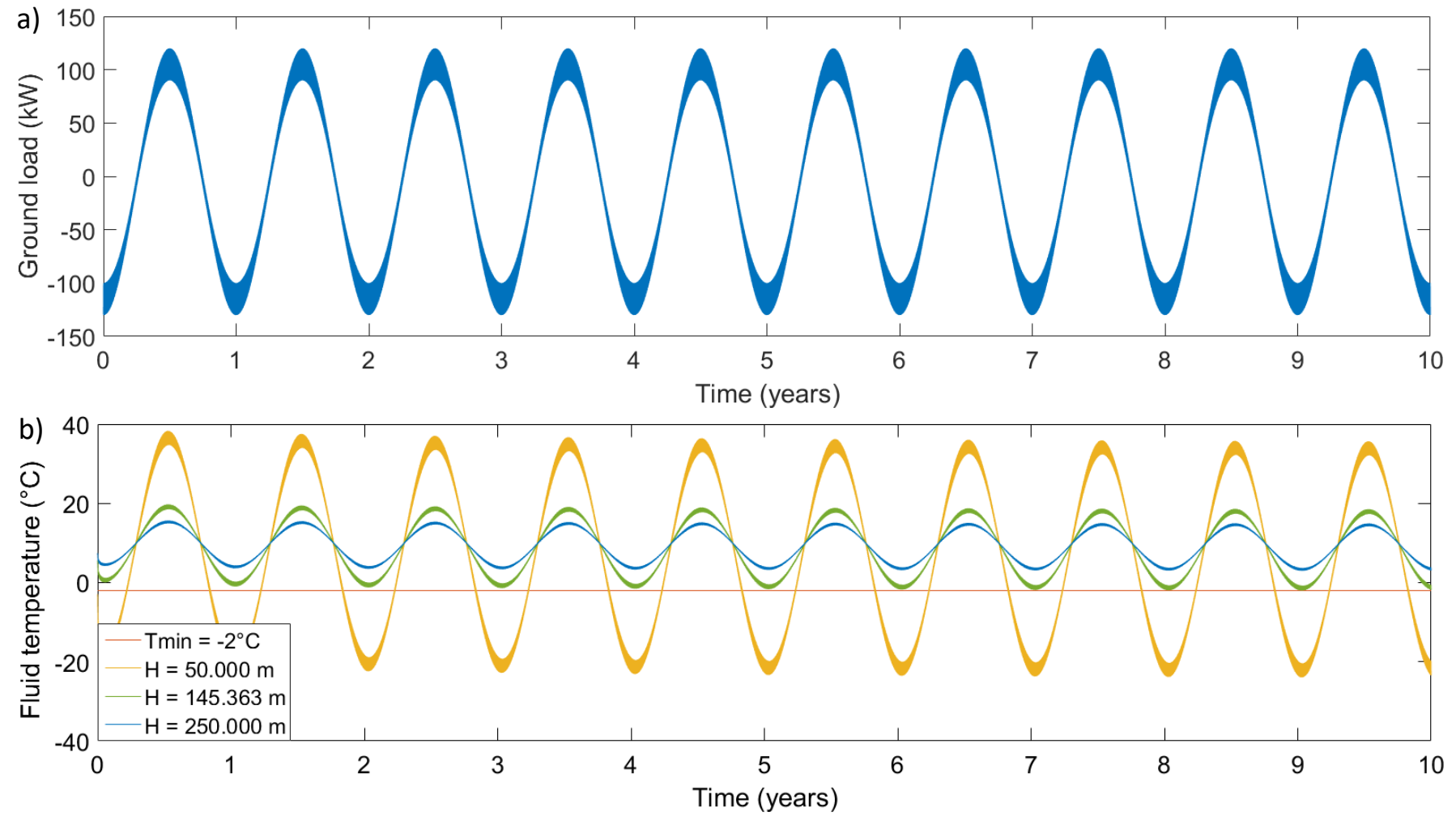

Figure 1 : a) Ground loads used for solution of Eq. 1 and b) evolution of fluid temperature for different borehole lengths.

\section{The objective function and its gradient as a function of borehole length}

Figure 2a below presents a sweeping of the possible values of $F$ and $d F / d H$ for $H$ values comprised between $30 \mathrm{~m}$ to $300 \mathrm{~m}$. We can see that these functions vary by several orders of magnitude with a clear minimum around $H=146 \mathrm{~m}$. Figure $2 \mathrm{~b}$ also illustrates easily the discrepancies that exists between the gradient computed by finite differentiation and the gradient computed with an analytical or semi-analytical approach.

When looking at Figure 2b, one can see that smaller differences also exist between the analytical and semianalytical methods. The magnitude of these discrepancies is grasped easily when looking at Table 1 which illustrates $F, d F / d H$ and the associated computation time for two different $H$ values. The first value was chosen to be as close as possible from the optimum of $F(145.363)$ while the second $H$ value $(250 \mathrm{~m})$ corresponds to a poor design. As shown in Table 1, the objective function values are equal for a given $H$. This is due to the fact that no matter which method is used for the calculation of the gradient, the objective function is always obtained the same way using the sequential method based on Marcotte and Pasquier (2014). However, the gradients computed by the analyticallybased methods are up to 30\% higher. For all six scenarios, 100 runs were completed to get a proper evaluation of the calculation times of the gradients by all three methods. As shown in Table 1, the analytical derivation method is systematically faster than the other two by at least $2 \%$ with our current MATLAB-based code. This is due to the shear fact that equivalent calculations that do not involved epsilons are compiled faster in our code. 

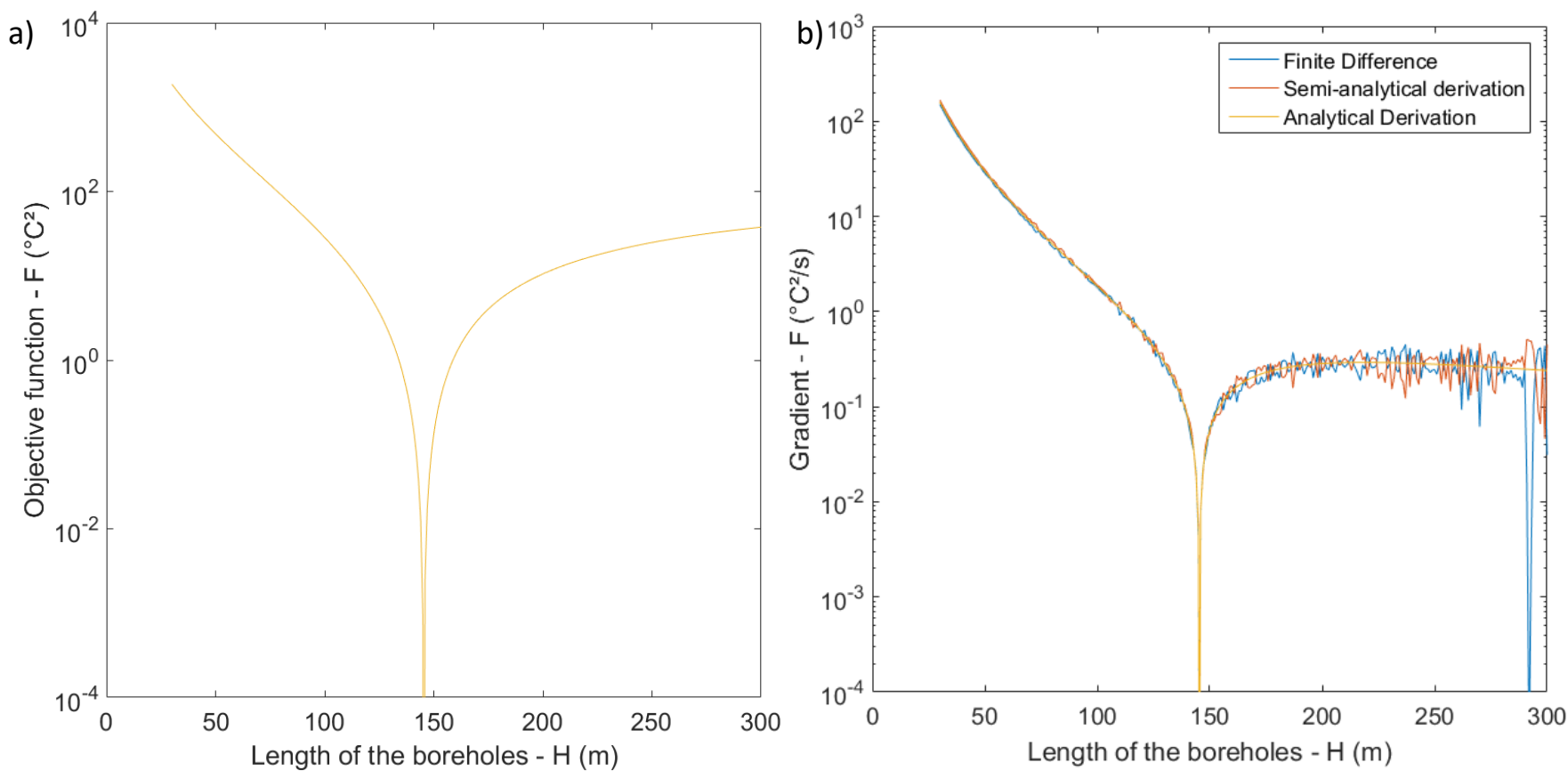

Figure 2: a) Objective function $F$ for various borehole lengths and b) gradients evaluated with the methods tested in this work with $\varepsilon=10^{-6}$.

Table 1. Objective Function Gradient for $H=145.363$ and $250.000 \mathrm{~m}\left(\varepsilon=10^{-6}\right)$

\begin{tabular}{ccccc}
\hline Method Used & $H(\mathbf{m})$ & $\begin{array}{c}\text { Objective Function } \\
\left({ }^{\circ} \mathbf{C}^{2}\right)\end{array}$ & $\begin{array}{c}\text { Gradient Value } \\
\left({ }^{\circ} \mathbf{C}^{2} / \mathbf{m}\right)\end{array}$ & $\begin{array}{c}\text { Computation Time } \\
(\mathbf{s})\end{array}$ \\
\hline \hline Finite Difference & 145.363 & $7.835 \mathrm{E}^{-10}$ & $-5.94 \mathrm{E}^{-6}$ & 0.386 \\
Semi-analytical Derivation & 145.363 & $7.835 \mathrm{E}^{-10}$ & $-4.141 \mathrm{E}^{-6}$ & 0.400 \\
Analytical Derivation & 145.363 & $7.835 \mathrm{E}^{-10}$ & $-4.621 \mathrm{E}^{-6}$ & 0.369 \\
Finite Difference & 250.000 & 24.809 & 0.2445 & 0.379 \\
Semi-analytical Derivation & 250.000 & 24.809 & 0.3149 & 0.399 \\
Analytical Derivation & 250.000 & 24.809 & 0.2797 & 0.372 \\
\hline
\end{tabular}

\section{OPTIMIZATION-BASED DESIGN USING A LINE SEARCH STRATEGY}

An accurate estimation of gradient is almost useless when used without an optimization algorithm able to minimize the objective function. Therefore, to illustrate the advantages of using analytical gradients for optimizationbased design, the various gradients were integrated within an optimization routine. Here, since the design problem involves only $H$, the problem reduces to a unidimensional line search. A line search algorithm using the strong Wolfe conditions on curvature, as implemented in minFunc (Schmidt, 2005), was used to identify the near-optimum $H$ value.

Table 2 and Table 3 provide the results for two different initial solutions of $H$ using a $\varepsilon$ value of 10-6 and 10-10 respectively. The optimization algorithm was limited to 5024 iterations to limit computation time, although it didn't prove to be necessary. All presented values of $H$ were limited to seven digits.

Looking at the results in both Table 2 and Table 3, three conclusions can be drawn. First, although the convergence rate all methods are influenced by $\mathrm{H}_{0}$, when the initial guess is too high compared to the optimal value, only the analytical-based derivation methods converge every time. Secondly, both the methods that implies a finite differentiation varies significantly in number of iterations depending on starting conditions. This is due to the fact that the convergence of these two methods relies heavily on the $\varepsilon$ value selected. This is especially true when the gradient to be evaluated is low and steady, associated with high initial values of $\mathrm{H}$ according to figure $2 \mathrm{~b}$, and when the epsilon 
is small. This implies that either a convergence analysis must be done or a safe low initial guess must be used in the optimization for the finite difference and semi-analytical methods in this particular case.

Thirdly, both the proposed analytical and the semi-analytical derivation methods converge way quicker than the finite difference method and in a lower number of iterations. On average, the semi-analytical method is 1.75 times faster while the analytical derivation method is 2 times faster. This is due to the fact than using a more precise gradient of the objective function allows for a much more rapid convergence. Indeed, Figure 3 illustrates the $H$ values used during the line search for the methods presented earlier and one can easily see the advantage of using gradients derived analytically.

Figure $1 \mathrm{~b}$ shows the simulated fluid temperature for $H$ equals to $50,145.363$ and $250 \mathrm{~m}$. The horizontal red line underneath the fluid temperature corresponds to the minimum fluid temperature of $-2^{\circ} \mathrm{C}$ that should be reached by the fluid to minimize the objective function $F$. It is clear that for $\mathrm{H}$ values of 50 and $250 \mathrm{~m}$, the fluid temperature is far from $-2^{\circ} \mathrm{C}$. However, for $\mathrm{H}$ corresponding to the optimum, the minimum occurs during the last winter and matches almost perfectly the red line.

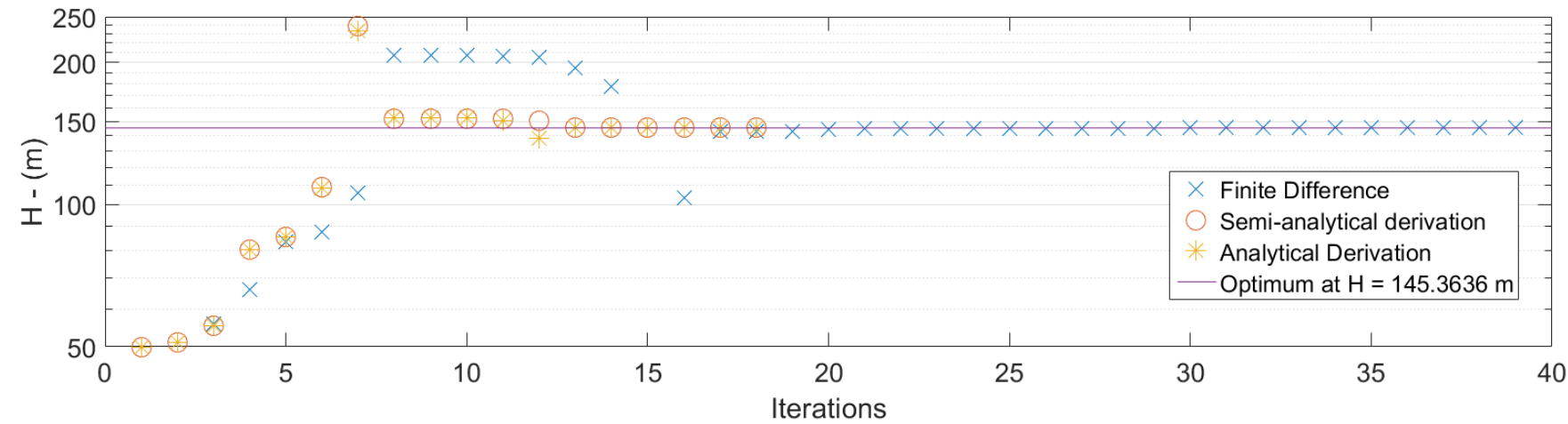

Figure 3 - Evolution of $H$ during the optimization-based design; initial guess $H=50 \mathrm{~m}, \varepsilon=10^{-6}$.

Overall, both analytical-based methods proved to be much faster and effective compared to the finite difference. The analytical derivation is more precise and allows for the fastest convergence and lowest number of iterations but requires more implementation time since it needs the calculation of a derived version of the FLS model and associated matrix. The semi-analytical method is almost as easy to implement as the common finite difference while allowing for a much faster rate of convergence. However, since it still requires to perform a finite difference on the transfer function of the GHE, the semi-analytical approach still requires the user to select more carefully its initial $H$ solution and epsilon before launching the optimization process.

Table 2. Line Search Results for Two Initial Solutions with $\varepsilon=10^{-6}$.

\begin{tabular}{|c|c|c|c|c|c|}
\hline Method Used & $\begin{array}{c}\text { Initial solution } \\
H_{0}(\mathrm{~m})\end{array}$ & $H(\mathrm{~m})$ & $\begin{array}{c}\text { Objective Function } \\
\left({ }^{\circ} \mathrm{C}^{2}\right)\end{array}$ & Iterations & $\begin{array}{r}\text { Computation } \\
\text { Time (s) } \\
\end{array}$ \\
\hline Finite Difference & 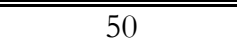 & 145.3626 & $3.58 \mathrm{E}^{-9}$ & 39 & 21.94 \\
\hline Analytical Derivation & 50 & 145.3636 & $4.07 \mathrm{E}^{-10}$ & 18 & 9.93 \\
\hline Finite Difference & 250 & 145.7234 & $1.07 \mathrm{E}^{-12}$ & 29 & 16.16 \\
\hline Semi-analytical Derivation & 250 & 145.3655 & $3.03 \mathrm{E}^{-8}$ & 15 & 8.72 \\
\hline
\end{tabular}


Table 3. Line search results for two initial solutions with $\varepsilon=10^{-10}$.

\begin{tabular}{|c|c|c|c|c|c|}
\hline Method Used & $\begin{array}{c}\text { Initial solution } \\
\qquad H_{0}(\mathrm{~m})\end{array}$ & $H(\mathrm{~m})$ & $\begin{array}{l}\text { Objective Function } \\
\qquad\left({ }^{\circ} \mathrm{C}^{2}\right)\end{array}$ & Iterations & $\begin{array}{r}\text { Computation } \\
\text { Time (s) }\end{array}$ \\
\hline Finite Difference & 50 & $\overline{51.0672}$ & 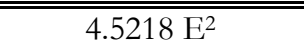 & 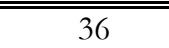 & 20.82 \\
\hline Semi-analytical Derivation & 50 & 145.3637 & $9.69 \mathrm{E}^{-10}$ & 23 & 14.10 \\
\hline Analytical Derivation & 50 & 145.3636 & $4.07 \mathrm{E}^{-10}$ & 18 & 9.93 \\
\hline Finite Difference & 250 & - & \multicolumn{3}{|c|}{ Did not converge within 5 minutes } \\
\hline Semi-analytical Derivation & 250 & 145.3647 & $9.99 \mathrm{E}^{-9}$ & 40 & 24.54 \\
\hline
\end{tabular}

\section{CONCLUSION}

In this article, we demonstrated that using a finite-difference based algorithms to evaluate the gradient of an objective function can lead to some approximation errors. When using such gradient in an optimization algorithm, even a simple unidimensional line search problem may have convergence issues or longer convergence time. By deriving analytically an objective function with respect to the design parameter of interest, a formulation that provides an accurate estimation of the gradient can be found. Using an analytical gradient in conjunction with strong Wolfe conditions may reduce computation time significantly.

\section{NOMENCLATURE}

$D \quad$ : Buried depth of the boreholes (m)

$\tilde{f}, f:$ Step increment vector $(\mathrm{W} / \mathrm{m})$

F : Objective function $\left({ }^{\circ} \mathrm{C}^{2}\right)$

$\widetilde{g}, g$ : Analytical model response $\left({ }^{\circ} \mathrm{C} \cdot \mathrm{m} / \mathrm{W}\right)$

G : Matrix whose element $(i, j)$ is the temperature perturbation caused at borehole $i$ by the heat emitted by borehole $j\left({ }^{\circ} \mathrm{C}\right)$

h : Vector containing the temperature perturbations due to the historical part $\left({ }^{\circ} \mathrm{C}\right)$

$H \quad$ : Length of the boreholes (m)

k : Thermal conductivity of the ground $\left(\mathrm{W} /\left(\mathrm{m} \cdot{ }^{\circ} \mathrm{C}\right)\right)$

$n_{b} \quad$ : Number of boreholes in the GHE (-)

$\mathbf{q}_{\mathbf{t}} \quad$ : Vector of heat flux $(\mathrm{W} / \mathrm{m})$
$\dot{Q}$ : Total ground load exchanged by the GHE (W)

$r$ : Distance between boreholes $i$ and $j(\mathrm{~m})$

$R_{b}$ : Borehole equivalent thermal resistance $\left(\mathrm{m} \cdot{ }^{\circ} \mathrm{C} / \mathrm{W}\right)$

$t \quad$ : Time (s)

$\bar{T}_{f}$ : Mean fluid temperature $\left({ }^{\circ} \mathrm{C}\right)$

$T_{g}$ : Initial temperature of the ground $\left({ }^{\circ} \mathrm{C}\right)$

$T_{\min }$ : Minimum fluid temperature $\left({ }^{\circ} \mathrm{C}\right)$

$\alpha \quad$ : Thermal diffusivity $\left(\mathrm{m}^{2} / \mathrm{s}\right)$

$\Delta \widetilde{T}_{b}:$ Transfer function corresponding to the mean temperature at the borehole wall $\left({ }^{\circ} \mathrm{C} / \mathrm{W} /\right.$ borehole $)$

$\varepsilon \quad$ : Increment used to perform finite difference (m)

\section{Subscripts}

$\begin{array}{ll}b & : \text { Borehole } \\ g & : \text { Ground } \\ i, j & : \text { Position } \\ m, t & : \text { Time step }\end{array}$




\section{REFERENCES}

Bernier M., Chahla A. and Pinel P. 2008. Long-Term Ground-Temperature Changes in Geo-Exchange Systems. ASHRAE Transactions. 114 (2): 342-350.

Cimmino M., Bernier M. and Adams F. 2013. A contribution towards the determination of g-functions using the finite line source. Applied Thermal Engineering. 51 (1-2): 401-412.

Claesson, J. and Javed S. 2011. An analytical method to calculate borehole fluid temperatures for time-scales from minutes to decades. ASHRAE Transactions. 117 (6): 279-288.

Eskilson P. 1987. Thermal Analysis of heat extraction borehole. Lund, Sweden: University of Lund.

Gehlin S. 2002. Thermal response test method development and evaluation. Lulea, Sweden: Luleå Tekniska Universitet.

Hénault B., Pasquier P. and Kummert M. 2016. Financial optimization and design of hybrid ground-coupled heat pump systems. Applied Thermal Engineering. 93: 72-82.

Huang S., Ma Z. and Wang F. 2015. A multi-objective design optimization strategy for vertical ground heat exchangers. Energy and Buildings. 87: 233-242.

Kavanaugh S. P. and Rafferty K. 2014. Geothermal Heating and Cooling: Design of Ground-Source Heat Pump Systems. ASHRAE.

Lamarche L. 2009. A fast algorithm for the hourly simulations of ground-source heat pumps using arbitrary response factors. Renewable Energy. 34 (10): 2252-2258.

Lazzarotto A. 2014. A network-based methodology for the simulation of borehole beat storage systems. Renewable Energy. 62: 265-275.

Marcotte D. and Pasquier P. 2008. On the estimation of thermal resistance in borehole thermal conductivity test. Renewable Energy. 33 (11): 2407-2415.

Marcotte D. and Pasquier P. 2014. Unite-response function for ground heat exchanger with parallel, series or mixed borehole arrangement. Renewable Energy. 68: 14-18.

Pasquier P. and Marcotte D. 2013. Efficient computation of heat flux signals to ensure the reproduction of prescribed temperatures at several interacting heat sources. Applied Thermal Engineering. 59: 515-526.

Pasquier P., Marcotte D., Bernier M. and Kummert M. 2013. Simulation of ground-coupled heat pump systems using a spectral approach. Proceedings of BS2013: 13th Conference of International Building Performance Simulation Association, Chambéry, France: 2691-2698.

Petersen K. B. and Pedersen M. S. 2012. The Matrix Cookbook. Technical University of Denmark. 72 pages.

Philippe M. et al. 2010. Sizing Calculation Spreadsheet: Vertical Geothermal Borefields. ASHRAE. 52 (7): 20-28.

Retkowski W. and Thöming J. 2014. Thermoeconomic optimization of vertical ground-source heat pump systems through nonlinear integer programming. Applied Energy. 114: 492-503.

Sayyaadi H., Amlashi E. H. and Amidpour M. 2009. Multi-objective optimization of a vertical ground source heat pump using evolutionary algorithm. Energy Conversion and Management. 50 (8): 2035-2046.

Schmidt M. 2005. minFunc: Unconstrained differentiable multivariate optimization in Matlab. Retrieved on September $15^{\text {th }}, 2016$ from http://www.cs.ubc.ca/ schmidtm/Software/minFunc.html. 